\title{
How an ontology can infer knowledge to be used in product conceptual design
}

\author{
David Cebrian-Tarrason ${ }^{1}$ and Rosario Vidal ${ }^{2}$ \\ ${ }^{1}$ Engineering Design Group (GID) - Department of Mechanical Engineering and \\ Construction. Universitat Jaume I, Spain, dcebrian@uji.es \\ ${ }^{2}$ Engineering Design Group (GID) - Department of Mechanical Engineering and \\ Construction. Universitat Jaume I, Spain, vidal@emc.uji.es
}

\begin{abstract}
In the past years, great advances have been made in the development of ontologies applied to the field of engineering design, essentially in functional and structural models. OntoFaBeS is an ontology whose objective is to formalize the knowledge about a product in order to infer different structures of that product from functional requirements set by the user. Hence, an effective tool capable of assisting the designer in the rational design phase is created. OntoFaBeS does not only provide the product redesign, but also allows for the evaluation of the different design alternatives created. OntoFaBeS can also capture the knowledge obtained from queries. This knowledge may be represented later in a CAE system. In order to illustrate the operation of OntoFaBeS, a mechanical pencil is used as an application example. This allows for a thorough assessment of the ontology design, wherein a query proves the ontology's ability to infer knowledge.
\end{abstract}

Keywords: Ontology, conceptual design, FBS, OntoFaBeS

\section{Introduction}

The end goal of Engineering Design is the conceptual creation of an object, product, system or process that meets functional requirements in order to fulfill the customer's needs in a workable, economical, ecological and manufacturable way. The development of technologies based on artificial intelligence and CAE (Computer Assisted Engineering) has facilitated access to information related to the structure and form of objects, although design know-how used in the conceptual design phase remains hidden due to its subjective nature and implicitness [1].

The development of KBE (Knowledge-Based Engineering) systems is aimed at improving this aspect $[2,3]$. Nonetheless, the full use of these technologies is limited by the impossibility of fully reusing and sharing knowledge in KBE 
systems, along with the lack of common knowledge from which to create a knowledge base and the limited success of methodologies for the extraction of knowledge [4, 5].

In the field of engineering design, more and more attention is being focused on the development of ontologies as a possible solution of the aforementioned deficiencies of KBE systems. This progress includes knowledge sharing and the development of a standard engineering language. One development of particular interest is to provide a structured basis for navigating, browsing and searching information through the hierarchical descriptions of the ontology $[6,7]$.

An ontology can be described as an explicit specification of a shared conceptualization, which can be taxonomically or axiomatically based [8]. Ontologies can be based around a single taxonomy or several taxonomies and their relationships [9]. Taxonomies consist of concepts and relationships that are organized hierarchically and whose concepts can be arranged as classes with subclasses $[10,11]$.

The structure of an ontology should be based on a taxonomy that allows for the modeling of a system based one certain functional descriptions [12]. In this way, a great diversity of methodologies for analysis of design process based on the FBS (Function-Behavior-Structure) framework have been modeled [13-16].

Notwithstanding, Suh [17] describes design as a zigzag between functional requirements and design parameters and Veyrat [18] questions whether design is actually a direct mapping between functions and structure. In order to resolve this question, we combine the concept of the OntoRFB (ontologically clean Functional Basis), Garbacz [12] based on DOLCE (Descriptive Ontology for Linguistic and Cognitive Engineering) [19] and the model B-FES (Behavior-driven FunctionEnvironment-Structure) $[20,21]$ and create a new ontology with special emphasis on the importance of behavior as the link in the FBS framework.

The purpose of this paper is the design of an ontology for the formalization of knowledge about a product, in order to infer different structures of that product from functional requirements set by the user, with the objective that knowledge can be reused and shared between different applications.

The paper is organized as follows. The next section briefly overviews the different approaches to the FBS framework. On the basis of the ontology that is being proposed, ontologies for Engineering Design are discussed in detail in section 3. Section 4 broadly describes the FBS framework of OntoFaBeS and a skeletal plant of the structure of the OntoFaBeS ontology. In order to illustrate the function of OntoFaBeS, a mechanical pencil is used as an example in section 5. Section 6 discusses this work, followed by concluding remarks. 


\section{FBS framework}

Though the terms function, behavior and structure had been used before, it wasn't until 1990 that they were clarified and used to define a framework to model and represent system functionality [13, 22]. In the FBS framework (FunctionBehavior-Structure), function represents the functions that the system performs; structure represents the physical elements of the solution and behavior acts as the relationship between $\mathrm{F}$ and $\mathrm{S}$. In design synthesis, behavior is derived from an intended functionality in order to reach a solution from it. When a solution is defined, its behavior is deduced from it to evaluate if the solution reaches the intended functionality. Then, behavior is related to the design physical state, which may be static or variable with time.

The FBS framework can also be used as a methodology for analyzing the design process, through the representation of the evolution of design state from the analysis of design protocols [23].

Roughly speaking, two approaches to FBS can be distinguished [24]. In the first, one relates functions to behaviors of an element, and then relates these behaviors to structural-physical descriptions of the elements. In the second approach, one models functions of objects in terms of inputs and outputs, and then relates these functions directly to structural-physical descriptions of objects (e.g. [25]).

The first approach was developed by Gero [22], who proposed a FunctionBehavior-Structure model of designing, and by Umeda et al. [13], who proposed a Function-Behavior-State model of designing. The second approach is known as the Functional Modeling approach which considers behavior as the mathematical representation of the states of a device [14, 26-28].

We will focus on the first approach since it considers behavior as a key concept that suggests a clear ontological ordering: technical objects have their physical structure. This structure, in interaction with a physical environment, gives way to the objects' behaviors; and these behaviors then determine in some way the objects' functions [15].

Several investigations have been developed with respect to this approach. Mizoguchi et al. [1] use the FBRL (Function and Behavior Representation Language) model based in the work of Sasajima et al. [29] and expresses behavior as a conceptualization of the change of attribute values in the spatio-temporal space over time. Also, they consider that function is a teleological interpretation of behavior under a given goal.

The possibilities of search, exploration, combination and selection systems based on FBS representation have increased thanks to the B-FES model proposed by Tor et al. [21, 30, 31]. This model is an extension and a refinement of the dualstep function environment-behavior-structure (FEBS) modeling framework by 
Deng et al. [32]. This model has been chosen by the authors because it maintains a clear ordering, which permits the establishment of direct relationships between function, behavior and design. However, structure is only considered on a superficial level.

After reviewing the above research efforts, it is evident that there is no integrated approach to modeling and a lack of a stable conceptual framework [33]. This is occurs mainly with respect to the concept of behavior. For example, Vermaas [34] indicates that the Functional Modeling approach does not manage to achieve an efficient relationship between those structures whose behavior is necessary in order to link with the function area. In the same way, in these situations behavior is partially subjective because depends on the designer [15]. Borgo et al. [15] formalizes behavior in order to consider it as 'a relationship between the object and the event in which it participates'. This analysis is carried out within the framework of the DOLCE ontology [19].

\section{Ontologies in Engineering Design}

In this section, we briefly discuss ontologies in the domain of engineering design. Generally, ontologies can be categorized by the subject of the conceptualization [35], among others:

Top-level ontologies or Upper-level ontologies describe very general concepts (e.g. substance, tangible, intangible) and provide general notions under which all root terms in existing ontologies should be linked.

Domain ontologies are reusable in a given specific domain (engineering, manufacturing, design, etc). These ontologies provide vocabularies about concepts within a domain and their relationships, the activities taking place in that domain, and the theories and elementary principles governing that domain.

Task ontologies describe the vocabulary related to a generic task or activity (e.g. diagnosing, scheduling). They provide a systematic vocabulary of the terms used to solve problems associated with tasks that may or may no belong to the same domain.

Application ontologies are application-dependent. They contain all the definitions needed to model the knowledge required for a particular application. Application ontologies often extend and specialize the vocabulary of the domain and of task ontologies for a given application.

In the domain of engineering design, such ontologies can be categorized into task ontologies and domain ontologies. As one of the principal ontologies of the Semantic Web, DOLCE is an upper-level ontology related to the area of engineering design. Thus, the goal of DOLCE is to provide a common reference framework for ontologies in order to facilitate sharing of information among them. 
In its representation, DOLCE aims at capturing ontological categories underlying natural language and human common-sense [19]. Thus, OntoFaBeS uses DOLCE as a common upper-level ontology in order to alleviate the integration problem. This kind of action is a frequent approach in ontological engineering [7].

Based on an extensive study of different design theories, Garbacz [12] puts forward the OntoRFB, a taxonomy of artifact functions based on DOLCE and on RFB [36], analyzed from a perspective of philosophical logic. Table 1 illustrates the actions of OntoRFB taxonomy.

Table 1 Actions of OntoRFB taxonomy.

\begin{tabular}{lllll}
\hline & Spatial Location & \multicolumn{2}{l}{$\begin{array}{l}\text { Topological } \\
\text { conectedness }\end{array}$} & Energy \\
\hline Quale region & Locate & Connect & Branch & Energate \\
Achieve & Reach & Touch & Split & Switch \\
Accomplish & Channel & Attach & Disjoin & Load \\
Mantain & Moor & Join & Cleave & Conserve \\
Process & Move & Bind & Carve & Energize \\
\hline
\end{tabular}

Gero, who improved his former study of FBS representation, establishes the basis for the computational modeling of process to support the design process based on the FBS representation [37]. He also developed the term "FBS Ontology" to refer to his model, although he did not develop a taxonomy, taking into account its own definition.

Engineering Design Integrated Taxonomy (EDIT) [8] consists of several taxonomies and their relations. As the integrated taxonomy is populated with instances, the relationships between concepts (or multiple concepts) are captured and the ontology emerges. Ahmed developed the EDIT ontology for the purpose of indexing, searching and retrieving design knowledge.

The DO (Design Ontology) is related to this work. Storga et al. [38] created the $\mathrm{DO}$ as a potential formal description of the shared engineering knowledge in design domain. Along these lines, Ahmed and DO created a comparison between both, where the DO is described as ontology conceived to describe design as a product. On the other hand, the EDIT was established design as an activity, incorporating the process as well as the product [8].

The majority of design ontologies that have been reviewed were mainly designed to facilitate knowledge sharing through well-defined ontological definitions. Applicability of ontologies for specific applications either remains unknown or they demonstrate their findings with very simple examples also known as "toy examples" [33]. That is, those consulted were not based on empirical research and therefore it is not possible to assess whether the ontology or taxonomy would be intuitive when used to index design knowledge [8]. The ontology developed by Kitamura et al. [39-41] is the only one that manages to develop a domain ontology and apply it to a practical example by means of the 
SOFAST ${ }^{\circledR}$ program. However, the complexity of Kitamura's model makes its practical application difficult [41].

There exists a great diversity of languages for ontology representation, although the most frequently used is the OWL (Ontology Web Language). Nonetheless, the majority of ontologies developed in this field do not make use of this standard language. OWL language allows for the use of logic, particularly in the form of predicate logic (also known as first-order logic), which allows for the inference of new knowledge by means of a query based on reasoning [12]. However, only the AsD ontology [42] makes use of this application to the area of manufacturing, and is also the only one to link the results of the ontology with a CAD program.

In conclusion, current ontologies in engineering design have certain failures. The lack of a diverse application of the concept of ontology within the FBS framework, the lack of practical examples on an industry level, the lack of homogeneity or the superficial application of the capacities of an ontology are all issues to consider. In the following section, by means of OntoFaBeS, we will propose an attempt to resolve these problems.

\section{OntoFaBeS}

In this section, we introduce OntoFaBeS, an ontology which formalizes the knowledge about a product in order to infer different structures of that product from functional requirements set by the user, with the objective that knowledge is reused and shared between different applications.

OntoFaBeS is a domain ontology partly based on the B-FES modeling framework $[21,30]$. It has been adapted to facilitate its modeling as an ontology. OntoFaBeS acquires its formal framework from the primitive notions defined in the DOLCE ontology [19], developed using the Protégé tool [43] and written in OWL, the standard ontology language.

The characteristics of OntoFaBeS have been defined with respect to its framework, function layer, behavior layer, structure layer and knowledge inference.

\section{Framework}

First, the connections between function and behavior were established, and then the connections between behavior and structure (Figure 1). The objective was to create a simple distribution. In part, this allowed for the construction of the taxonomy, while also facilitating the creation of logical rules upon the creation of the ontology.

\section{Function Layer}

The definition of the concept function (F) was established as the inherent action of the design that is being processed. As a result, actions carried out by elements as independent units outside of the design environment were excluded (e.g. the 
action carried out by a screw in an object is not a function). In this ontology design, the possibility of defining functions with natural language was considered, as well as the option than an object could have more than one function.

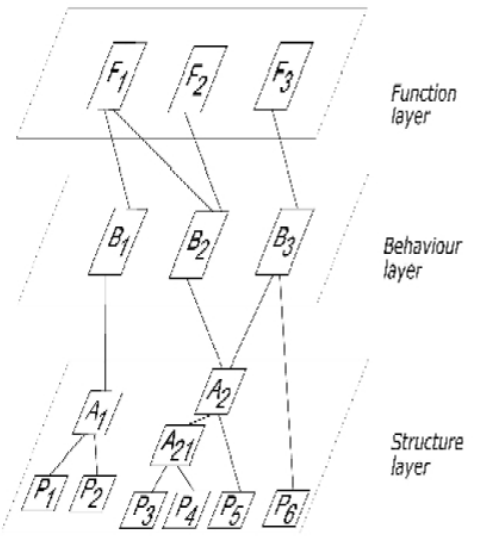

Figure 1 OntoFaBeS framework.

\section{Behavior Layer}

A new perspective was established, based on the definition made by Borgo [16], by considering the actions in Table 1 as behaviors. The behavior (B) is tied to an action with respect to an element. In the aforementioned taxonomy, the proposed classification of actions is always linked to an element constituent of part of the structure.

\section{Structure Layer}

A layer dedicated exclusively to structure was considered. It was organized in such a way that the elements of the structure phase could be linked with each other and with the behavior layer, taking into account the difference between parts $(\mathrm{P})$ and assemblies (A). Since the proposed model could be made up of a large number of elements, the bases of modular design [44] were incorporated in order to optimize the assembly phase.

Thus, each part of an object, as part of a structure, has an implicit relationship with other parts or assemblies.

\section{Knowledge Inference}

Once the content is stored in the ontology, OntoFaBeS could capture the knowledge obtained from queries in order to directly relate the functions with the structure that would fulfill the requirements. This would allow the structures resulting from a java application to be represented later in a CAE system through an interface, after giving the program the necessary parameters to define the dimension of the parts. From the relationships created by the ontology, we can determine which elements could constitute the assemblies and their corresponding parts. 
In this way, OntoFaBeS achieves an integrated approach to the FBS model due to its clarity, solid structure and simplicity. The use of a limited table of behaviors results in a detailed analysis of the knowledge that makes up the design. Also, it allows for the use of the logical bases of an ontology, by contemplating the possibility of inference. This creates great flexibility for considering different options, not contemplated or directly restricted.

\section{Mechanical Pencil}

A practical example has been considered in order to demonstrate the capacity of the use of an ontology as a base for translating the FBS scheme. It is the same example as previously employed by Gershenson [45]. It consists of a basic example, of which a detailed list of functions, behavior and structure is available.

A mechanical pencil is a metallic, wooden or plastic instrument that contains leads and is used for writing. It consists of a mechanism made up of four modules: Sleeve/Tip, Clutch/Teeth, Barrel and Cap. (Figure 2)

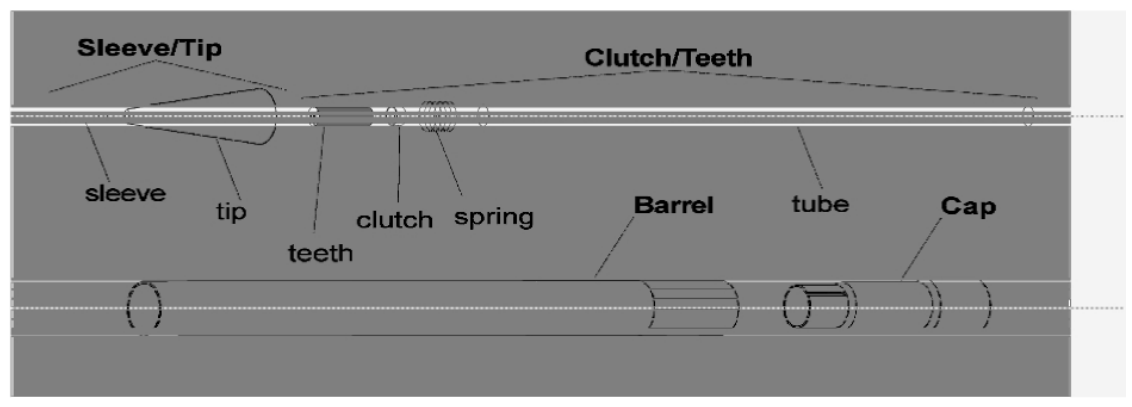

Figure 2 Mechanical Pencil.

The pencil is thus made up of the following elements: barrel, cap, clutch, sleeve, spring, tip, teeth and tube.

By establishing the FBS scheme for this example, we find that the function of the pencil is: write. According to the organization established by OntoRFB taxonomy [12], the behavior of the mechanical pencil with respect to the function write is to carve. We must also consider that the constituent elements are made up by the aforementioned four modules.

Each module of the example has the following behaviors:

- Barrel Assembly: locate (contains the leads within the pencil) and touch (allows for the pencil to be held)

- Pencil mechanism (Clutch/Teeth Assembly): attach (holds the lead) and channel (it channels the lead out of the pencil). 
- Spring: move (by offering resistance and allowing for the gradual advance of the pencil lead)

- Clutch: touch (allows for the pencil to be held)

○ Teeth: attach (holds the lead)

- Tube: moor (supports the lead so that it can be propelled by the spring)

- Cap: branch (allows the clutch to be propelled forward).

- Pencil tip (Sleeve/Tip Assembly): attach (holds the lead) and reach (the lead reaches the paper)

- Pencil lead ${ }^{1}$ : carve (it erodes upon contact with the paper)

A behavior that describes the union of all the elements is connect, which establishes relationships between the elements that make up an assembly (e.g. cone connects spring). This lets us establish one of the essential logical rules established by the ontology, which will allow for the inference of knowledge.

These are the behaviors that allow the mechanical pencil to fulfill its function, to write. Nonetheless, these behaviors when linked to other elements can, in turn, lead to other functions. For example the tube, with different dimensions can be used in another design to conduct water, maintaining the same behavior but linking to completely different functions. These other functions can be fulfilled by other elements, which is how the inference of information is carried out by OntoFaBeS.

These are the behaviors that allow the mechanical pencil to fulfill its function, to write, since with the knowledge acquired by the ontology, it is concluded that the main part is the pencil lead and it must be connected to the next assemblies: cone/tip, clutch/teeth, barrel and cap.

Nonetheless, a behavior when is linked to other elements of a design can, in turn, link to completely different functions maintaining the same behavior. These other functions can be carried out with the help of other elements, which is how the inference of information is carried out by OntoFaBeS. For example the tube, with different dimensions can be used in another product design as a pipe. In that case, it would maintain the same behavior, to moor, but would be linking to the function to channel water. Thus, the product would also need a water pump in order to fulfill that function.

Finally, a query can be carried out from which the user can determine directly the indicated structure (Figure 3).

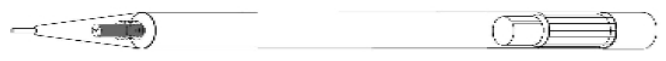

Figure 3 Mechanical Pencil inferred.

\footnotetext{
${ }^{1}$ The pencil lead has not been considered as a constituent of the pencil, since it is a consumable.
} 


\section{Conclusion}

We have introduced OntoFaBeS as an intent to unify the existing criteria in the field of methodologies based on the FBS framework. This study illustrates the design of an ontology formally based on the B-FES [30] and whose formal framework is based on the upper ontology DOLCE [19], providing a novel approach that is centered on design behaviors.

This work opens up a new perspective in the field of design engineering methodologies. OntoFaBeS takes advantage of the formalization of knowledge in ontologies, that is, the evolution of knowledge based engineering [4, 45]. This results in a series of advantages that until now had not been dealt with in depth in the field of engineering design, such as the automatic inference of new knowledge.

It is interesting to note that OntoFaBeS deduces the knowledge necessary for the production of objects, based exclusively on the requirements established by the consumer. This also demonstrates that the great majority of ontologies existing in the field of engineering design are essentially taxonomies that give little importance to formal logic and the possible inference of new knowledge.

The results of this study are not surprising however, if we take into account that OntoFaBeS contemplates a novel focus on behavior within the FBS framework. Nonetheless, a great deal of knowledge is necessary on behalf of the designer in order to establish the relationships correctly. For this reason, the improvement of OntoFaBeS is under development. Also, a wider scope of actions is being considered, as well as a deeper analysis of the development of the functional layer.

The proposed example of a mechanical pencil demonstrates the importance of the appropriate definition of the three layers that constitute the FBS framework when it comes to constructing the ontology. This allows for the successful establishment of the information queries. This is especially in the behavior area, due to its role as the link between function and structure. It is important to note that the simplicity of the example was intentional, as the initial application of a new concept.

In future investigations, we intend to apply this model on an industrial level by means of its use on an existing design. Work is also being carried out to develop the ontology in order to apply it to areas outside of engineering design. At this time, OntoFaBeS is being adapted to encompass other aspects of the design phase, such as environmental aspects.

\section{References}

1.Kitamura Y., Mizoguchi R.: Ontology-based systematization of functional knowledge. Journal of Engineering Design, 15/4, pp. 327-351 (2004).

2.Cebrian-Tarrason D., Muñoz C., Chulvi V., Vidal R.: Nuevo Enfoque En El Diseño Inteligente De Implantes Craneales Personalizados A Través De KBE. XI Congress International of Project Engineering, Lugo, Spain (2007). 
3.Chulvi V., Sancho A., Cebrian-Tarrason D., Jiménez R., Muñoz C., Vidal R.: KnowledgeBased Engineering in cranioplasty implant design. Proceedings of the 16th International Conference on Engineering Design (ICED'07), Paris (2007).

4.Mizoguchi R.: Tutorial on ontological engineering. New Generation Computing, Part 1, pp. 365-384 (2003).

5.Baxter D., Gao J., Case K., Harding J., Young B.: An engineering design knowledge reuse methodology using process modeling. In: Research in engineering design, pp. 18, 37-48 (2007).

6.Ahmed S., Wallace K.: Reusing Design Knowledge. 14th CIRP Design Seminar, Cairo (2004).

7.Ahmed S., Kim S., Wallace K.: A methodology for creating ontologies for engineering design. Journal of computing and information science in engineering, 7/2, pp. 132-140 (2006).

8.Gruber T.R.: Toward Principles for the Design of Ontologies Used for Knowledge Sharing, K.A. Publishers. In Knowledge Systems Laboratory, Stanford University, Padova, Italy (1993).

9.Cottam H, Milton N., Shadbolt N.: The Use of Ontologies in a Decision Support System for Business Process Re-engineering, Information Technology and Knowledge Re-Engineering. Journal of the Austrian Computing Society, Vienna, Budapest (1998).

10.Gilchrist A.: Thesauri, taxonomies and ontologies - an etymological note. Journal of Documentation. 59/1, pp. 7-18.

11.Antoniou G., Harmelen F.: A semantic web primer, The MIT Press, Cambridge, Massachusetts (2003).

12.Garbacz P.: Towards a standard taxonomy of artifact functions. Applied Ontology, 1/3, pp. 221-236.

13.Umeda Y., Takeda H.: Function behavior, and structure. Applications of Artificial Intelligence in Engineering, vol. V, pp. 177-194 (1990).

14.Wood K.L., Greer J.L.: Function-Based Synthesis. In Methods in Engineering Design, pp. 170-227 (2001).

15.Borgo S., Carrara M., Vermaas P.E., Garbacz P.: Behavior of a technical artifact: An ontological perspective in engineering. Frontiers in Artificial Intelligence and Applications. 150 (Formal Ontology in Information Systems), pp. 214-225 (2006).

16.Umeda Y., Takeda H.: Development of design methodology for upgradable products based on function-behavior-state modeling. Artificial Intelligence for Engineering Design. Analysis and Manufacturing (AI EDAM), vol. 19, pp. 161-182 (2005).

17.Suh N.P.: The principles of design, Oxford University Press, New York (1990).

18.Veyrat N., Blanco E., Trompette P.: When Shape Does Not Induce Function: Why Designers Must Not Lose The Big Picture. 16th International Conference on Engineering Design, Paris (2007).

19.Masolo C.: WonderWeb Deliverable D18. Laboratory For Applied Ontology - ISTC-CNR. (2003).

20.Zhang W.Y., Tor S.B., Britton G.A.: FuncDesigner a functional design software system. The International Journal of Advanced Manufacturing, vol. 22, pp. 295-305, (2006).

21.Zhang W.Y., Tor S.B., Britton G.A.: A graph and matrix representation scheme for functional design of mechanical products. The International Journal of Advanced Manufacturing Technology, vol. 25(3-4), pp. 221-232 (2005).

22.Gero J.: Design prototypes: A knowledge representation schema for design. AI magazine. Vol. 11(4), pp. 26-36 (1990).

23.Takeda H., Yoshioka M., Tomiyama M., Shimomura Y.: Analysis of design processes by function, behavior and structure. The Delft Protocols Workshop, conference proceedings (1994). 24.Camelo D.: Modelado y desarrollo de un modelo computacional de síntesis interactivo y multirrelacional para guiar la actividad de diseño en la fase conceptual. $\mathrm{PhD}$ Thesis. University Jaume I (2007).

25.Chandrasekaran B.: Representing function: Relating functional representation and functional modeling research streams. AIEDAM. vol. 19(2), pp. 65-74 (2005).

26.Pahl G., Beitz W.: Engineering design. In Design Council (1984).

27.Pahl G., Beitz W.: Engineering Design. A Systematic Approach. London, Springer (1996). 
28.Szykman S., Racz J., Sriram R.: The representation of function in computer-based design. Design Engineering Technical Conferences, ASME, Las Vegas, Nevada (1999).

29.Sasajima M., Kitamura Y., Ikeda M., Mizoguchi R.: FBRL: A Function and Behavior Representation Language. Proc. of IJCAI-95. pp. 1830-1836 (1995).

30.Tor S.B., Zhang W.Y., Britton G.A.: Guiding functional design of mechanical products through rule-based causal behavioural reasoning. International Journal of Production Research. vol. 40(3), pp. 667-682 (2002).

31.Tor S.B., Britton G.A, Zhang W.Y.: A knowledge-based blackboard framework for stamping process planning in progressive die design. The International Journal of Advanced Manufacturing Technology, vol. 26, pp. $774-783$ (2005).

32.Deng Y.M.: A computerized design environment for functional modeling of mechanical products. Fifth ACM symposium on Solid modeling and applications. ACM Press, Ann Arbor, Michigan (1999).

33.Vermaas P.E., Dorst K.: On the conceptual framework of John Gero's FBS-model and the prescriptive aims of design methodology. Design Studies. vol. 28(2), pp.133-157 (2007).

34.Vermaas P.E.: The Functional Modelling Account of Stone and Wood: Some Critical Remarks. Proceedings of the 16th International Conference on Engineering Design, Paris (2007). 35.Gómez-Pérez A., Fernández-López M., Corcho O.: Ontological Engineering with examples from the areas of Knowledge Management, e-Commerce and the Semantic Web. Springer (2004).

36.Hirtz J., Stone R.B., McAdams D.A., Szykman S., Wood K.L.: A functional basis for engineering design: Reconciling and evolving previous efforts. Research in Engineering Design, vol. 13, pp. 65-82 (2002).

37.Gero J.S. and Kannengiesser U.: The situated function-behavior-structure framework. Design Studies, vol. 25(4), pp. 373-391 2004.

38.Štorga M., Andreasen M.M., Marjanović D.: Towards a formal design model based on a genetic design model system. Proceedings of the 15th International Conference on Engineering Design ICED 05. Melbourne, Australia (2005).

39.Kitamura Y., Mizoguchi R.: An ontology-based annotation framework for representing the functionality of engineering devices. In ASME 2006, Pennsylvania, USA (2006).

40.Kitamura Y. Mizoguchi R., Ontology-based description of functional design knowledge and its use in a functional way server. Expert Systems with Applications, vol. 24(2), pp. 153-166 (2003).

41.Ookubo M., Koji Y., Sasajima M., Kitamura Y., Mizoguchi R.: Towards Interoperability Between Functional Taxonomies Using An Ontology-Based Mapping, Proceedings of the 16th International Conference on Engineering Design, Paris (2007).

42.Kim, K.-Y., Manley, D.G., Yang, H.: Ontology-based assembly design and information sharing for collaborative product development. Computer-Aided Design, vol. 38(12) pp. 1233 1250 (2006).

43.Protégé 3.3.1., http://protégé.Stanford.edu. Stanford University. (2008).

44.Gershenson, J.K., Prasad, G.J., Allamneni, S.: Modular Product Design: A Life-Cycle View. Journal of Integrated Design \& Process Science, vol. 3, pp.13-26 (1999).

45.Gero, J.S.: AI EDAM at 20: Artificial intelligence in designing, Cambridge Univ Press (2007) 